\title{
Effects of chest compressions on cardiovascular and cerebral hemodynamics in asphyxiated near-term lambs
}

\author{
Kristina S. Sobotka', Graeme R. Polglase ${ }^{1,2}$, Georg M. Schmölzer ${ }^{3,4}$, Peter G. Davis ${ }^{4}$, Claus Klingenberg ${ }^{5,6}$ and Stuart B. Hooper ${ }^{1,2}$
}

\begin{abstract}
BACKGROUND: Chest compressions (CC) and adrenaline administration are recommended in asphyxiated newborns with persistent bradycardia despite effective ventilation. The effects of CC on cerebral blood flow in newborns at birth are unknown. Our aim was to determine the effects of CC, with or without adrenaline administration, on the return of spontaneous circulation, carotid blood flow (CBF), and carotid arterial pressure (CAP) in asphyxiated near-term lambs.
\end{abstract}

METHODS: Asphyxia was induced in near-term lambs by clamping the umbilical cord and delaying ventilation onset until spontaneous circulation ceased. Lambs were then resuscitated by positive pressure ventilation along with CC followed by adrenaline administration. CAP and CBF were continuously recorded.

RESULTS: Mean CAP did not increase significantly during CC and only increased following adrenaline administration. CC did not increase mean CBF but increased CBF amplitude due to increased peak flow and the onset of retrograde flow during diastole. Adrenaline increased mean CBF from $1 \pm 2$ to $15 \pm 5 \mathrm{ml} / \mathrm{kg} / \mathrm{min}$ and abolished retrograde diastolic CBF, leading to the return in spontaneous circulation.

CONCLUSION: We conclude that CC with adrenaline administration was required to increase CBF and restore spontaneous circulation in asphyxiated lambs. Low CBF and retrograde diastolic CBF during CC indicate hypoperfusion to the brain.

A pproximately $5 \%$ of all newborns worldwide will require some form of assisted ventilation at birth and about $0.03 \%$ of newborns will require more advanced resuscitation such as intubation, chest compressions (CCs), and drug administration $(1,2)$. Severely asphyxiated newborn infants are born bradycardic and apneic and require resuscitation to establish pulmonary gas exchange and restore cardiac function after birth. As the initiation of ventilation and the establishment of a functional capacity also trigger the increase in pulmonary blood flow at birth, it plays a dual role in severely asphyxiated newborn infants (3). That is, it is responsible for both increasing oxygenation and for facilitating the increase in cardiac output by providing preload for the left ventricle $(3,4)$. If low cardiac output persists despite effective positive pressure ventilation, the newborn is likely to suffer increasingly severe hypoxia and acidemia, leading to hypoxic/ischemic injury. Continued asphyxia further depresses myocardial function, leading to low systolic and diastolic blood pressures despite a chemoreceptor-mediated peripheral vasoconstriction $(5,6)$. Current international neonatal resuscitation guidelines recommend that if heart rate is less than 60 BPM despite effective positive pressure ventilation, CCs should be applied $(7,8)$. CC will assist to mechanically pump blood until the myocardium is sufficiently oxygenated to recover spontaneous function (9). If heart rate remains below $60 \mathrm{BPM}$ despite effective $\mathrm{CC}$, then adrenaline should be administered $(7,8)$.

Given the infrequent use of $\mathrm{CC}$ and adrenaline, rigorous clinical studies to optimize CC and adrenaline administration are difficult to complete. Consequently, the evidence base for these interventions during neonatal resuscitation remains low, and data is extrapolated largely from adult or animal resuscitation studies $(1,7,8)$. However, most animal studies have been conducted in adult models $(10,11)$ or newborn animals (12-14) that were hours or days into postnatal life. Therefore, most of the information from these studies is of limited applicability to neonatal delivery room resuscitation. In particular, they do not take into consideration all of the unique changes that occur during the transition from fetal to newborn life which are complex and multifactorial. For instance, newborn infants will initially have airways that are filled with liquid, which prevents pulmonary gas exchange, the pulmonary circulation will be vasoconstricted, and the ductus arteriosus (DA) will continue to shunt the majority of right ventricular output into the descending aorta. All of these factors will influence the ability to effectively resuscitate severely asphyxiated infants.

The aim of this study was to determine the effects of CC prior to and following adrenaline administration on the return of spontaneous circulation and on carotid hemodynamics in asphyxiated near-term lambs immediately after birth.

\section{RESULTS}

Uninterrupted CCs were performed at a compression to ventilation ratio of 2.2:1 and continued for a median time of $135 \mathrm{~s}$ 
(Table 1). CC rate remained constant for the duration of CC (Figure 1). Adrenaline was administered $58 \pm 34 \mathrm{~s}$ (mean \pm SEM) after CC was initiated, and spontaneous circulation was restored $60 \pm 17 \mathrm{~s}$ after administration of adrenaline.

Mean heart rate before CC was $72 \pm 7$ BPM (Figure 1). Heart rate increased to $115 \pm 12$ with the onset of CC. After the completion of CC during circulatory recovery, heart rate increased further to $180 \pm 19 \mathrm{BPM}$.

Mean carotid arterial pressure (CAP) before initiation of $\mathrm{CC}$ was $9.3 \pm 2.4 \mathrm{~mm} \mathrm{Hg}$ (Figure 2a). Mean CAP increased to $12.7 \pm 3.7 \mathrm{~mm} \mathrm{Hg}$ with the initiation of $\mathrm{CC}$, but this was not significant. Mean CAP increased significantly to $21.5 \pm 6.8$ $\mathrm{mm} \mathrm{Hg}$ after adrenaline was administered. Immediately after adrenaline administration, mean CAP increased further to $52.4 \pm 3.9 \mathrm{~mm} \mathrm{Hg}$ and continued to increase until it stabilized at $71.7 \pm 4.6 \mathrm{~mm} \mathrm{Hg}$. Peak systolic CAP increased twofold with the initiation of CC, increasing further with the administration of adrenaline and during circulatory recovery (Figure 2b). Interestingly, mean diastolic CAP was $6.6 \pm 1.1$ before initiation of CC and did not increase when CCs were performed alone (Figure 2c). Mean diastolic CAP began to increase $20 \mathrm{~s}$ after adrenaline was administered and increased to $38.8 \pm 3.4$ $\mathrm{mm} \mathrm{Hg}$ by the end of CC and increased further during circulatory recovery.

Mean carotid blood flow (CBF) before CC was $0.94 \pm 0.72 \mathrm{ml} /$ $\mathrm{kg} / \mathrm{min}$ (Figure 3a). Following the initiation of CC, the

Table 1. Characteristics of CCs and adrenaline administration from the onset of ventilation

\begin{tabular}{lc}
\hline Parameters & Values \\
\hline Ratio of compressions to ventilation & $2.2: 1$ \\
Duration of asphyxia before ventilation onset (min) & $10.5(7-13.5)$ \\
Initiation of CC from ventilation onset (s) & $227.5(102-344)$ \\
Duration of CC (s) & $135(43-144)$ \\
Time of adrenaline administration from start of CC (s) & $24(0-213)$ \\
Time of circulatory recovery from adrenaline & $45(7-124)$ \\
administration (s) & \\
\hline
\end{tabular}

Data are median (range).

CC, chest compression.

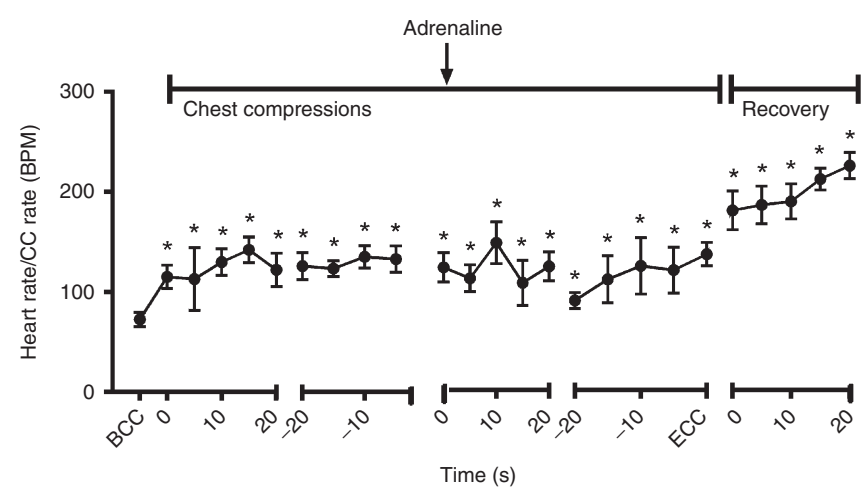

Figure 1. Heart rate or chest compression (CC) rate in asphyxiated lambs before chest compressions (BCC), after initiation of CC and administration of adrenaline, end of chest compressions (ECC), and during recovery when spontaneous circulation returned. Data are mean \pm SEM. ${ }^{*} P<0.05$ vs. BCC. amplitude of the CBF waveform markedly increased (Figure 4), which resulted from both an increase in peak and a decrease in minimum CBF. Retrograde CBF (represented as a negative value) was greatest during early diastole and resulted in a negative mean diastolic CBF despite forward flow returning at end diastole (Figure 3c). Mean diastolic CBF remained negative until adrenaline was administered, whereupon it increased to $14.4 \pm 2.1 \mathrm{ml} / \mathrm{kg} / \mathrm{min}$ at the cessation of CC and then increased further to $30.1 \pm 6.5$ during circulatory recovery. This increase was mostly reflected by a loss of retrograde early-diastolic $\mathrm{CBF}$ in response to adrenaline administration. The high retrograde blood flow during diastole contributed to the unaltered mean CBF when CC was initiated (Figure 3c), despite a large increase in peak CBF. During CC, adrenaline administration
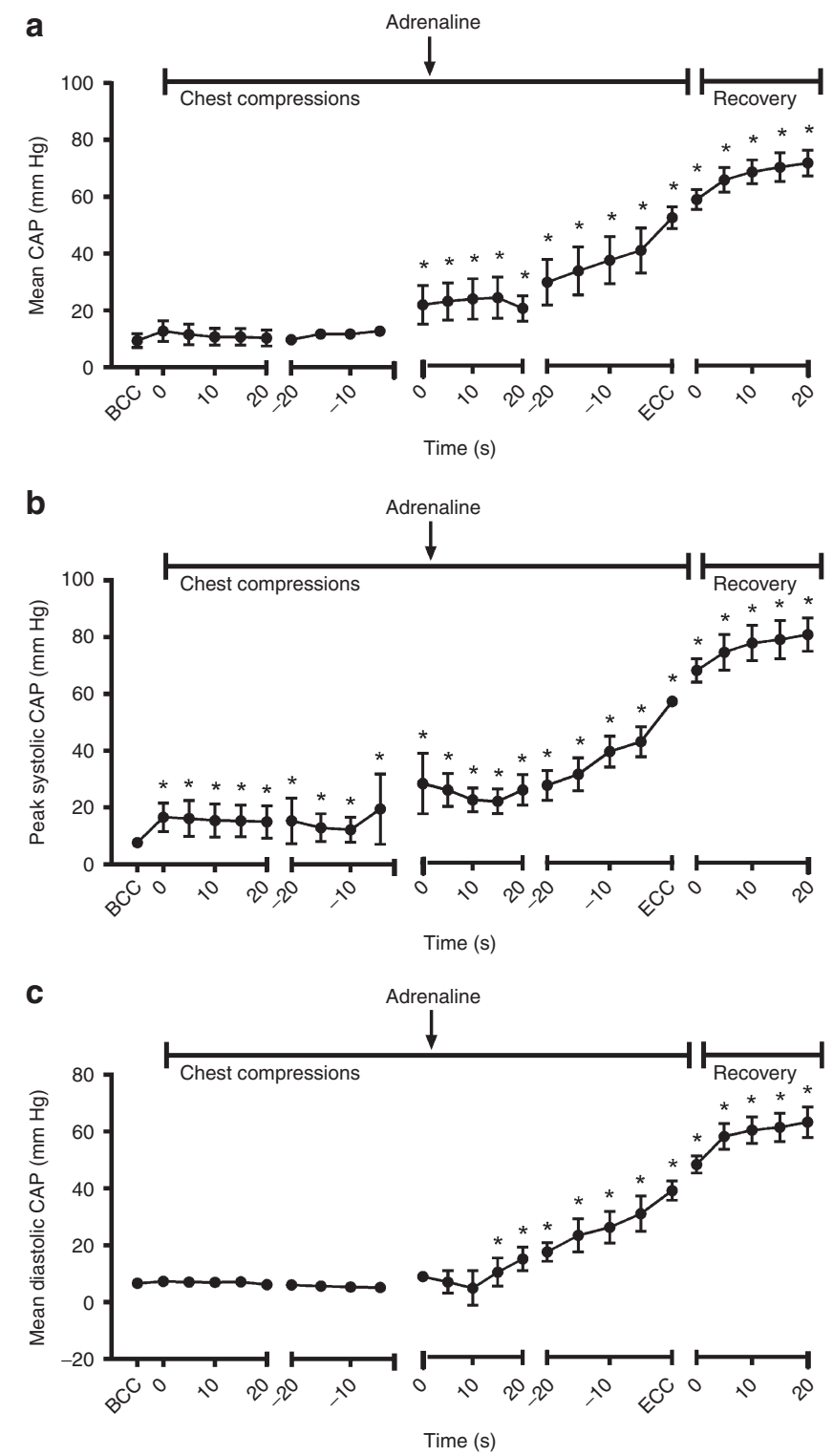

Figure 2. Carotid arterial blood pressure. (a) Mean carotid arterial pressure (CAP), (b) peak systolic CAP, and (c) mean diastolic CAP in asphyxiated lambs before chest compressions (BCC), after initiation of CC and administration of adrenaline, end of chest compressions (ECC), and during recovery when spontaneous circulation returned. Data are mean \pm SEM. ${ }^{*} P<0.05$ vs. BCC. 

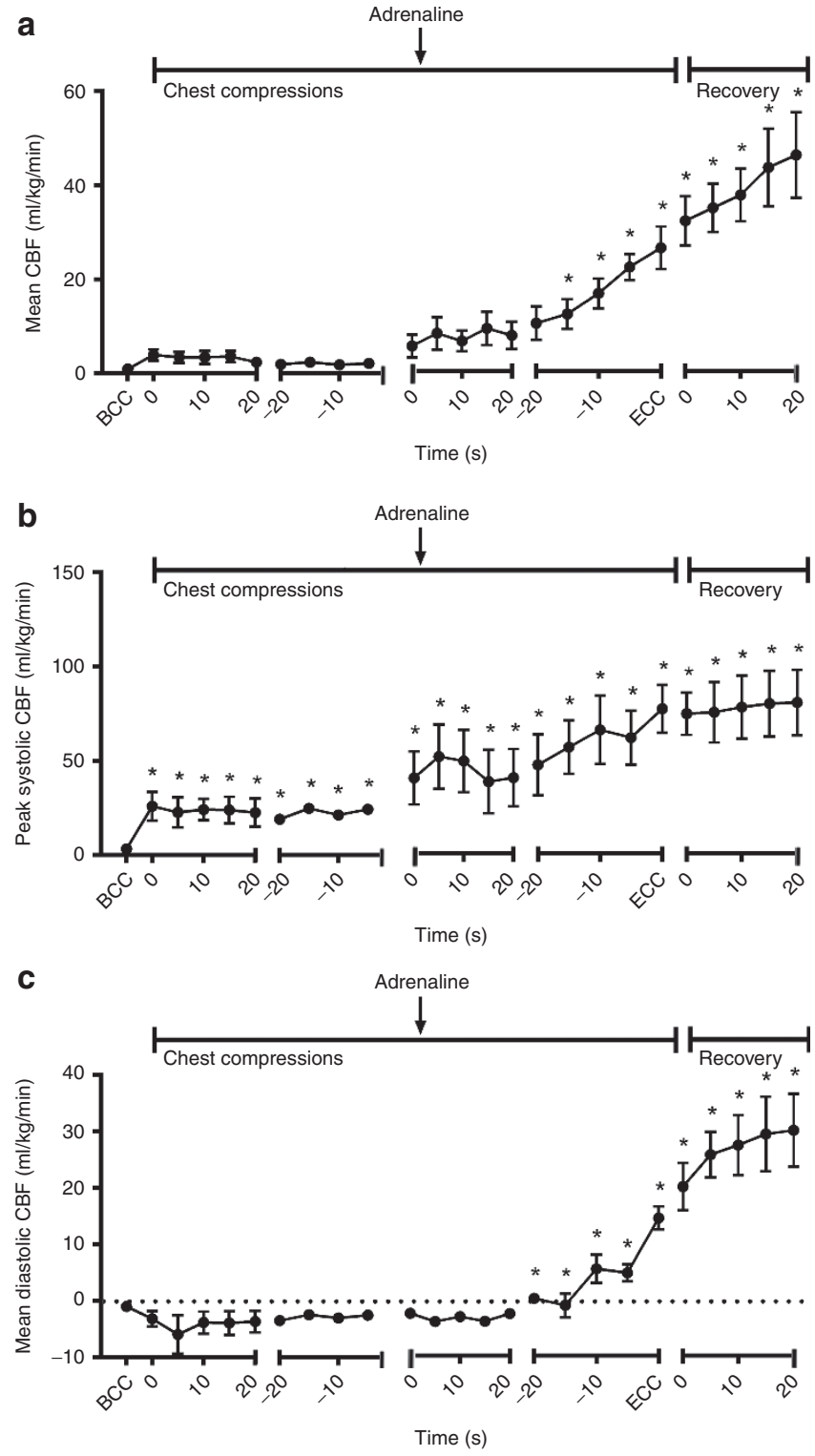

Figure 3. Carotid arterial blood flow. (a) Mean carotid arterial blood flow (CBF), (b) peak systolic CBF, and (c) mean diastolic CBF in asphyxiated lambs before chest compressions (BCC), after initiation of $C C$ and administration of adrenaline, end of chest compressions (ECC), and during recovery when spontaneous circulation returned. Data are mean \pm SEM. ${ }^{*} P<0.05$ vs. BCC.

increased mean CBF to $26.2 \pm 4.6 \mathrm{ml} / \mathrm{kg} / \mathrm{min}$ which increased further to $46 \pm 9.1 \mathrm{ml} / \mathrm{kg} / \mathrm{min}$ during circulatory recovery. Peak systolic CBF increased from $3.1 \pm 1.6 \mathrm{ml} / \mathrm{kg} / \mathrm{min}$ before $\mathrm{CC}$ to $25.7 \mathrm{ml} / \mathrm{kg} / \mathrm{min}$ after the initiation of $\mathrm{CC}$ and continued to increase to $40.2 \pm 14.2 \mathrm{ml} / \mathrm{kg} / \mathrm{min}$ after adrenaline administration (Figure $3 b$ ). Peak systolic CBF increased further to $74.5 \pm 11.3 \mathrm{ml} / \mathrm{kg} / \mathrm{min}$ during circulatory recovery.

\section{DISCUSSION}

Advanced resuscitation, including CCs at birth, is required to reestablish circulatory stability in about $0.03 \%$ of newborn infants. The findings from our study demonstrate that, following circulatory arrest, the combination of CC and conventional ventilation for up to $213 \mathrm{~s}$ was unable to restore spontaneous

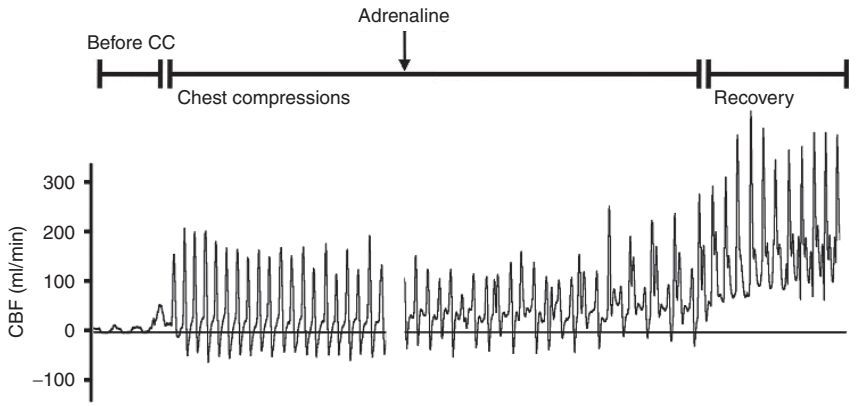

Figure 4. Example of the physiological recordings of carotid arterial blood flow waveforms before chest compressions (CCs), during CC and adrenaline administration, and recovery after CC.

circulation in asphyxiated lambs immediately after birth. Adrenaline was critical for increasing CAP and CBF and for restoring spontaneous circulation. We observed that although CCs were able to generate large peak CBF values, large retrograde flows occurred during early diastole, which resulted in little or no increase in mean CBF. However, following adrenaline administration, retrograde $\mathrm{CBF}$ ceased as diastolic pressures increased and, as a result, mean CBF markedly increased.

Numerous studies have previously investigated the optimum strategies for reestablishing spontaneous circulation in newborn animals using various cardiopulmonary resuscitation strategies (15-18). However, the relevance of these studies to the resuscitation of asphyxic infants at birth is limited because they were conducted in newborn animals at $12-36 \mathrm{~h}$ after birth. At this time, the airways are air-filled, not liquid-filled, and the circulation will have transitioned into the adult phenotype. As a result, the DA is likely to be functionally closed, pulmonary vascular resistance (PVR) will be low, the entire output of the right ventricle will flow through the lung, and little or no output from the left ventricle will shunt left to right through the DA and enter the pulmonary circulation. In contrast, at birth, the lungs must aerate to initiate pulmonary gas exchange, which leads to a large decrease in PVR, and pulmonary blood flow must increase to take over the role of umbilical venous return as the main source of preload for the left ventricle (4); left to right shunting through the DA contributes to this increase in PBF and preload for the left ventricle (3). Collectively, these changes reflect an enormous physiological transition in both the cardiovascular and respiratory systems, which must have a major impact on the ability of resuscitation strategies to revive infants at birth. To the best of our knowledge, our study is the first to investigate the influence of CC during this critical period of newborn life.

Following cessation of circulation, CCs were performed according to international resuscitation guidelines $(7,8)$. However, despite the initiation of CC, neither mean systemic arterial pressures nor $\mathrm{CBF}$ increased even when CC continued for more than $3 \mathrm{~min}$. We observed a rapid increase in peak systolic CAP and CBF when CC was initiated, which demonstrates that CCs are very effective at increasing stroke volume, but the large degree of retrograde flow counteracted the high peak flow, resulting in low mean flows. Furthermore, the 


\section{Articles | Sobotka et al.}

restoration of spontaneous cardiac function was found to be unrelated to the onset and duration of CC.

It is well established that following cardiac arrest in newborns, spontaneous circulation is only restored when diastolic blood pressure increases, which reestablishes coronary perfusion (19). In our study, mean and diastolic CAP and CBF increased only after adrenaline was administered. Adrenaline restores cardiac function by increasing cardiac contractility (via $\beta$-adrenergic receptors) and peripheral vasoconstriction (via $\alpha$-adrenergic receptors), consequently increasing venous return (20). It is unknown whether increased cardiac contractility or peripheral vasoconstriction has the greatest influence, although the peripheral vascular bed should be maximally vasoconstricted in response to the asphyxia. Nevertheless, our findings are consistent with the findings of previous studies investigating asphyxia-induced asystole in postnatal piglets $(16,17)$. They found that although increasing the compressions to ventilation ratios from 3:1 to 9:3 or 15:2 generated higher diastolic blood pressures, these higher pressures were still unable to restore spontaneous circulation, which only occurred after adrenaline administration $(16,17)$.

There is increasing evidence demonstrating the success of continuous uninterrupted CC performed on adults with cardiac arrest improves survival rate (21). However, it is important to note that CC-only cardiopulmonary resuscitation has not been shown to be more effective than conventional cardiopulmonary resuscitation in incidences of cardiac arrest of noncardiac origin (i.e., due to respiratory compromise such as drowning or asphyxia) (22). Adults that go into sudden cardiac arrest (e.g., from ventricular fibrillation) can have adequately oxygenated blood for the initial 4 to $6 \mathrm{~min}$ without ventilatory efforts (23). Therefore, uninterrupted CC would be beneficial by increasing coronary perfusion and supplying the heart with oxygenated blood. During asphyxia, the patient is already hypoxic and acidemic at the time of cardiac arrest, therefore effective ventilation is also important to recovery $(16,17)$. It is important to remember that the circulatory and ventilatory status of an asphyxiated newborn is different to some adult models of cardiac arrest, where they often have well-oxygenated blood before time of arrest.

During CC, the retrograde $\mathrm{CBF}$ we observed during diastole is unlikely to be due to left-to-right shunting through the DA (ductal steal) at least initially. This is because there was insufficient time for PVR to decrease enough to allow blood to flow predominantly from left to right through the DA. Retrograde blood flow during diastole in the carotid, cerebral, mesenteric, and renal arteries is commonly observed in preterm infants with a large patent DA (24-26). However, this can only occur when PVR is lower than downstream resistance in these peripheral vascular beds. Indeed, it can take many minutes for PVR to decrease after ventilation onset, although hypoxic vasoconstriction of the peripheral vascular bed will facilitate left to right shunting through the DA. Instead, we suggest that the loss of diastolic pressure reduces the critical blood volume within the large arteries below that required to maintain tension on the arterial walls. As a result, blood simply flows backwards and forwards during CC and indicates that diastolic pressure in the large proximal arteries is required to sustain forward flow in distal vascular beds, such as in the cerebral vascular bed. Unfortunately, no data were collected on blood flow through the DA in our study to determine the presence or magnitude of ductal steal. Nevertheless, it is likely that the end result will be similar to ductal steal, which causes systemic hypoperfusion and is associated with increased risks of intraventricular hemorrhage, pulmonary hemorrhage, necrotizing enterocolitis, and renal failure (27).

Although peak systolic CAP markedly increased in lambs during CC, diastolic CAP remained unaffected indicating reduced cardiac function in the asphyxiated lamb as it was unable to increase diastolic pressure, despite the assistance of CC. Perinatal asphyxia is associated with the development of myocardial dysfunction resulting in a reduction in cardiac output and mean blood pressure (28). This loss in cardiac function likely explains the reduction in diastolic blood pressure, which in turn explains the retrograde $\mathrm{CBF}$ during diastole as explained above.

Rapid abnormal fluctuation to cerebral blood flow is a major mechanism of brain injury in newborn preterm infants (29). We observed alarmingly large fluctuations in CBF during CC. Fluctuations in cerebral blood flow following an ischemic insult increases the likelihood of cerebral vessel rupture and hemorrhaging in the neonatal brain $(30,31)$. Asphyxia also impairs cerebral autoregulation, so the brain cannot maintain cerebral blood flow at a near steady state, leaving the brain more vulnerable to cardiovascular hemodynamic instability and hemorrhagic injury $(32,33)$. Therefore, these rapid fluctuations to cerebral blood flow may partially explain why infants given cardiopulmonary resuscitation have poor neurological outcomes and increased risk of developing grade 3 or 4 intraventricular hemorrhages (34).

Interestingly, we found that heart rate was a poor indicator of circulatory status in these asphyxiated lambs. Despite a mean heart rate of $72 \pm 7 \mathrm{BPM}$ (well above the $60 \mathrm{BPM}$ threshold outlined in the International Liaison Committee on Resuscitation guidelines (7)), mean CBF was zero, and the systolic peak in flow was barely detectable (Figure 3). This indicates that heart rate should be cautiously used to assess circulatory status in newborn asphyxiated infants.

In conclusion, we have described for the first time the effect of CC and adrenaline administration on blood flow to the brain. We have demonstrated that $\mathrm{CC}$ alone was not enough to restore spontaneous circulation in asphyxiated newborn lambs and that adrenaline administration was critical for increasing heart rate, CAP, and CBF. CCs alone resulted in large fluctuations in peak systolic CAP and CBF that are potentially injurious, along with retrograde diastolic $\mathrm{CBF}$ that greatly reduced mean CBF. This retrograde flow persisted until adrenaline was administered, which rapidly restored arterial pressures and forward flow during diastole. It is likely that these responses may lead to cerebral vascular injury and hemorrhaging, particularly in asphyxiated infants who are likely to have maximally dilated their cerebral vascular bed in response to the asphyxia. Given that this study was a subgroup of a larger experimental 
group, and not the original aim of the study, these conclusions need to be taken with caution. More rigorous studies need to be undertaken before results are taken to a clinical setting. However, this is a first step in addressing the need for evidence-based resuscitation studies using more relevant models of neonatal asphyxia.

\section{METHODS}

All experimental procedures were approved by the relevant Monash University Animal Ethics Committee. The data were collected and analyzed from lambs that were enrolled in a previously published study (35). However, the lambs reported in the current study required CCs and adrenaline to restore cardiac function following the asphyxia and so were not included in our previous study. Pregnant ewes at $139 \pm 4$ (mean \pm SD) d of gestation (term is $\sim 147 \mathrm{~d}$ ) were anesthetized, and the fetal head and neck were exposed via cesarean section. Catheters were inserted into a fetal carotid artery and jugular vein. An ultrasonic flow probe (3PS; Transonic Systems, Ithaca, NY) was placed around the noncatheterized carotid artery, as previously described (36). The fetal trachea was intubated with a cuffed endotracheal tube $(5 \mathrm{~mm})$, lung liquid was drained passively for $20 \mathrm{~s}$, and then, the endotracheal tube was clamped. A transcutaneous pulse oximeter (Masimo, Irvine, CA) was attached around the right forelimb before the umbilical cord was clamped and cut. Lambs were delivered, dried, weighed, and placed under a radiant heater (Fisher \& Paykel Healthcare, Auckland, New Zealand). Initial asphyxia was induced in the lamb by delaying initiation of ventilation until carotid mean arterial pressure had decreased to $\sim 20 \mathrm{~mm} \mathrm{Hg}$, at which time the endotracheal tube was opened and ventilation commenced. Following the asphyxia period, ventilation was initiated (Babylog 8000+ ventilator; Dräger, Lübeck, Germany) using pressure limited ventilation $\left(35 \mathrm{cmH}_{2} \mathrm{O}\right.$, positive end-expiratory pressure $5 \mathrm{cmH}_{2} \mathrm{O}$, inspiratory time $0.5 \mathrm{~s}$, and expiratory time 0.5 s). Despite ventilation onset, if flow in the carotid artery ceased and mean arterial pressures were below $10 \mathrm{~mm} \mathrm{Hg}$, CCs were initiated. CCs were performed by three clinicians (G.M.S., P.D., and C.K.) using the two-thumb encircling technique. The anterior to posterior chest depth was measured prior to the experiment in each lamb and CC using a depth of $1 / 3$ of the depth of at least $1 / 3$ of the diameter of the anterior to posterior chest depth. As the chest of a lamb is V-shaped, it is different to a human chest, but piglets and lambs have been used previously to study physiological changes during neonatal CC. In addition, as thoracic pump theory suggests that CC acts by increasing intrathoracic pressure, this suggests that CCs are feasible in lambs (37). It is important to note that despite carotid arterial blood flow ceasing, a heartbeat was still detectable (60-70 BPM), indicating that heart rate is not a good indicator of cardiac function in this model. CCs $(2.2: 1$ compression to ventilation ratio, Table 1) commenced as soon as flow in the carotid artery had ceased. Adrenaline (10-15 $\mu \mathrm{g} / \mathrm{kg}$ i.v.) was administered soon after (administration time ranged from CC onset to $213 \mathrm{~s}$ after, Table 1). Lambs received 5\% dextrose (i.v.), were sedated (alfaxane i.v.: $5-15 \mathrm{mg} / \mathrm{kg}$ /h; Jurox, East Tamaki, Auckland, New Zealand) and intubated. Ewes were killed (sodium pentobarbitone: $\sim 100 \mathrm{mg} / \mathrm{kg}$ i.v.) after delivery of the lamb.

CBF (Powerlab; ADInstruments, Castle Hill, New South Wales, Australia) and CAP (DTX Plus Transducer; Becton Dickinson, Singapore) were recorded continuously prior to delivery until the end of the experiment. We recorded the delivered tidal volume and pressure limited ventilation simultaneously with blood flow and pressure data. Heart rate was measured from the CAP wave.

\section{Analytical Methods}

Heart rate, CAP, and CBF in six lambs $(n=6)$ were averaged over five cardiac cycles immediately before CC, then every $5 \mathrm{~s}$ for $20 \mathrm{~s}$ after initiation of CC, $20 \mathrm{~s}$ before administration of adrenaline, 20 $\mathrm{s}$ before end of CC, and $20 \mathrm{~s}$ after completion of CC, and spontaneous circulation was restored (circulatory recovery). Circulatory recovery was determined when a spontaneous heartbeat was seen on the carotid arterial flow recordings and diastolic pressure was increasing.

\section{Statistical Methods}

Data were analyzed using one-way repeated measures ANOVA with time as a factor. Post hoc comparisons between time points before CCs and after initiation of CC were performed using the Holm-Sidak test. A $P<0.05$ was accepted as statistically significant. Data are presented as mean \pm SEM unless otherwise stated.

\section{ACKNOWLEDGMENTS}

We thank Karyn Rodgers for her assistance with these experiments.

\section{STATEMENT OF FINANCIAL SUPPORT}

This study was supported by National Health and Medical Research Council (NHMRC) of Australia program grant (No. 384100), fellowship (G.R.P.: 1026890, and S.B.H.: 545921), a Rebecca L. Cooper Medical Research Foundation Fellowship (G.R.P.), and the Victorian Government's Operational Infrastructure Support Program.

\section{Disclosure: None.}

\section{REFERENCES}

1. Vento M, Saugstad OD. Resuscitation of the term and preterm infant. Semin Fetal Neonatal Med 2010;15:216-22.

2. Li Z, Zeki R, Hilder L, Sullivan E. Australia’s Mothers and Babies 2011. Perinatal Statistics, Series No. 28. Canberra, Australia: AIHW National Perinatal Epidemiology and Statistics Unit, 2013.

3. Crossley KJ, Allison BJ, Polglase GR, Morley CJ, Davis PG, Hooper SB. Dynamic changes in the direction of blood flow through the ductus arteriosus at birth. J Physiol 2009;587(Pt 19):4695-704.

4. Bhatt S, Alison BJ, Wallace EM, et al. Delaying cord clamping until ventilation onset improves cardiovascular function at birth in preterm lambs. J Physiol 2013;591(Pt 8):2113-26.

5. Giussani DA, Spencer JA, Moore PJ, Bennet L, Hanson MA. Afferent and efferent components of the cardiovascular reflex responses to acute hypoxia in term fetal sheep. J Physiol 1993;461:431-49.

6. Wassink G, Bennet L, Booth LC, et al. The ontogeny of hemodynamic responses to prolonged umbilical cord occlusion in fetal sheep. J Appl Physiol (1985) 2007;103:1311-7.

7. Perlman JM, Wyllie J, Kattwinkel J, et al.; Neonatal Resuscitation Chapter Collaborators. Part 11: Neonatal resuscitation: 2010 International Consensus on Cardiopulmonary Resuscitation and Emergency Cardiovascular Care Science With Treatment Recommendations. Circulation 2010;122:Suppl 2:S516-38.

8. Kattwinkel J, Perlman JM, Aziz K, et al.; American Heart Association. Neonatal resuscitation: 2010 American Heart Association Guidelines for Cardiopulmonary Resuscitation and Emergency Cardiovascular Care. Pediatrics 2010;126:e1400-13.

9. Kapadia V, Wyckoff MH. Chest compressions for bradycardia or asystole in neonates. Clin Perinatol 2012;39:833-42.

10. Roberts JR, Greenberg MI, Knaub MA, Kendrick ZV, Baskin SI. Blood levels following intravenous and endotracheal epinephrine administration. JACEP 1979;8:53-6.

11. Mielke LL, Frank C, Lanzinger MJ, et al. Plasma catecholamine levels following tracheal and intravenous epinephrine administration in swine. Resuscitation 1998;36:187-92.

12. Berg RA, Otto CW, Kern KB, et al. A randomized, blinded trial of highdose epinephrine versus standard-dose epinephrine in a swine model of pediatric asphyxial cardiac arrest. Crit Care Med 1996;24:1695-700.

13. Berg RA, Hilwig RW, Kern KB, Ewy GA. "Bystander" chest compressions and assisted ventilation independently improve outcome from piglet asphyxial pulseless "cardiac arrest". Circulation 2000;101:1743-8.

14. Dean JM, Koehler RC, Schleien CL, et al. Improved blood flow during prolonged cardiopulmonary resuscitation with $30 \%$ duty cycle in infant pigs. Circulation 1991;84:896-904.

15. Dannevig I, Solevåg AL, Wyckoff M, Saugstad OD, Nakstad B. Delayed onset of cardiac compressions in cardiopulmonary resuscitation of newborn pigs with asphyctic cardiac arrest. Neonatology 2011;99:153-62.

16. Solevåg AL, Dannevig I, Wyckoff M, Saugstad OD, Nakstad B. Extended series of cardiac compressions during CPR in a swine model of perinatal asphyxia. Resuscitation 2010;81:1571-6. 


\section{Articles | Sobotka et al.}

17. Solevåg AL, Dannevig I, Wyckoff M, Saugstad OD, Nakstad B. Return of spontaneous circulation with a compression:ventilation ratio of $15: 2$ versus 3:1 in newborn pigs with cardiac arrest due to asphyxia. Arch Dis Child Fetal Neonatal Ed 2011;96:F417-21.

18. Solevåg AL, Dannevig I, Nakstad B, Saugstad OD. Resuscitation of severely asphyctic newborn pigs with cardiac arrest by using $21 \%$ or $100 \%$ oxygen. Neonatology 2010;98:64-72.

19. Wyckoff MH. Neonatal cardiopulmonary resuscitation: critical hemodynamics. NeoReviews 2010;11:e123-9.

20. Attaran RR, Ewy GA. Epinephrine in resuscitation: curse or cure? Future Cardiol 2010;6:473-82.

21. Hüpfl M, Selig HF, Nagele P. Chest-compression-only versus standard cardiopulmonary resuscitation: a meta-analysis. Lancet 2010;376:1552-7.

22. Kitamura T, Iwami T, Kawamura T, Nagao K, Tanaka H, Hiraide A; Implementation Working Group for All-Japan Utstein Registry of the Fire and Disaster Management Agency. Bystander-initiated rescue breathing for out-of-hospital cardiac arrests of noncardiac origin. Circulation 2010; 122:293-9.

23. Chandra NC, Gruben KG, Tsitlik JE, et al. Observations of ventilation during resuscitation in a canine model. Circulation 1994;90:3070-5.

24. Perlman JM, Hill A, Volpe JJ. The effect of patent ductus arteriosus on flow velocity in the anterior cerebral arteries: ductal steal in the premature newborn infant. J Pediatr 1981;99:767-71.

25. Serwer GA, Armstrong BE, Anderson PA. Continuous wave Doppler ultrasonographic quantitation of patent ductus arteriosus flow. J Pediatr 1982;100:297-9.

26. Lipman B, Serwer GA, Brazy JE. Abnormal cerebral hemodynamics in preterm infants with patent ductus arteriosus. Pediatrics 1982;69:778-81.

27. El-Khuffash A, Higgins M, Walsh K, Molloy EJ. Quantitative assessment of the degree of ductal steal using celiac artery blood flow to left ventricular output ratio in preterm infants. Neonatology 2008;93:206-12.
28. Van Bel F, Walther FJ. Myocardial dysfunction and cerebral blood flow velocity following birth asphyxia. Acta Paediatr Scand 1990;79:756-62.

29. Del Toro J, Louis PT, Goddard-Finegold J. Cerebrovascular regulation and neonatal brain injury. Pediatr Neurol 1991;7:3-12.

30. Perlman JM, McMenamin JB, Volpe JJ. Fluctuating cerebral blood-flow velocity in respiratory-distress syndrome. Relation to the development of intraventricular hemorrhage. N Engl J Med 1983;309:204-9.

31. Van Bel F, Van de Bor M, Stijnen T, Baan J, Ruys JH. Aetiological rôle of cerebral blood-flow alterations in development and extension of peri intraventricular haemorrhage. Dev Med Child Neurol 1987;29: 601-14.

32. Lou HC, Lassen NA, Friis-Hansen B. Impaired autoregulation of cerebral blood flow in the distressed newborn infant. J Pediatr 1979;94:118-21.

33. Lou HC, Lassen NA, Tweed WA, Johnson G, Jones M, Palahniuk RJ. Pressure passive cerebral blood flow and breakdown of the blood-brain barrier in experimental fetal asphyxia. Acta Paediatr Scand 1979;68: $57-63$.

34. Wyckoff MH, Salhab WA, Heyne RJ, Kendrick DE, Stoll BJ, Laptook AR; National Institute of Child Health and Human Development Neonatal Research Network. Outcome of extremely low birth weight infants who received delivery room cardiopulmonary resuscitation. J Pediatr 2012;160:239-244.e2.

35. Klingenberg C, Sobotka KS, Ong T, et al. Effect of sustained inflation duration; resuscitation of near-term asphyxiated lambs. Arch Dis Child Fetal Neonatal Ed 2013;98:F222-7.

36. Polglase GR, Wallace MJ, Grant DA, Hooper SB. Influence of fetal breathing movements on pulmonary hemodynamics in fetal sheep. Pediatr Res 2004;56:932-8.

37. Halperin HR, Tsitlik JE, Guerci AD, et al. Determinants of blood flow to vital organs during cardiopulmonary resuscitation in dogs. Circulation 1986;73:539-50. 